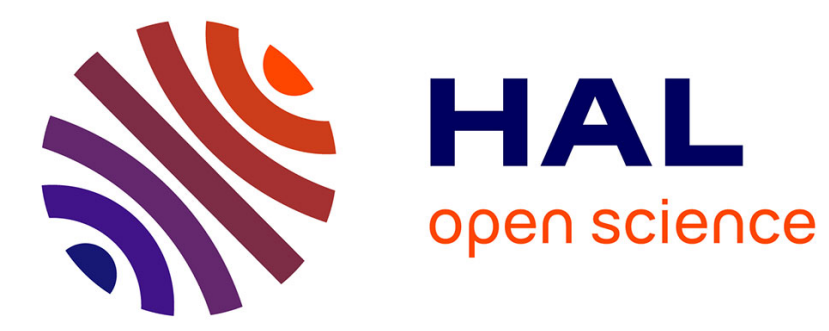

\title{
Influence des phénomènes de relaxation sur la forme des impulsions fournies par un laser $\mathrm{CO} 2$ déclenché par un absorbant saturable
}

\author{
J. Dupré, F. Meyer, C. Meyer
}

\section{> To cite this version:}

J. Dupré, F. Meyer, C. Meyer. Influence des phénomènes de relaxation sur la forme des impulsions fournies par un laser CO2 déclenché par un absorbant saturable. Revue de Physique Appliquée, 1975, 10 (5), pp.285-293. 10.1051/rphysap:01975001005028500 . jpa-00243914

HAL Id: jpa-00243914

https://hal.science/jpa-00243914

Submitted on 1 Jan 1975

HAL is a multi-disciplinary open access archive for the deposit and dissemination of scientific research documents, whether they are published or not. The documents may come from teaching and research institutions in France or abroad, or from public or private research centers.
L'archive ouverte pluridisciplinaire HAL, est destinée au dépôt et à la diffusion de documents scientifiques de niveau recherche, publiés ou non, émanant des établissements d'enseignement et de recherche français ou étrangers, des laboratoires publics ou privés. 


\title{
INFLUENCE DES PHÉNOMẼNES DE RELAXATION SUR LA FORME DES IMPULSIONS FOURNIES PAR UN LASER $\mathrm{CO}_{2}$ DÉCLENCHÉ PAR UN ABSORBANT SATURABLE
}

\author{
J. DUPRÉ, F. MEYER et C. MEYER \\ Laboratoire d'Infrarouge (*), Université de Paris VI, Bâtiment 350, 91405 Orsay, France
}

(Reçu le 25 novembre 1974, révisé le 3 mars 1975, accepté le 22 avril 1975)

\begin{abstract}
Résumé. - La forme des impulsions laser, fournies par un laser à gaz carbonique déclenché par un absorbant saturable, $\mathbf{S F}_{6}$, a été étudiée expérimentalement ; un modèle théorique du phénomène a été élaboré. Il permet de reconstituer un train d'impulsions laser. Dans les tests effectués sur cette méthode de calcul, l'accent a été mis particulièrement sur le rôle de la relaxation rotationnelle dans l'absorbant.
\end{abstract}

Abstract. - The shape of the pulses generated by a passive $Q$-switched $\mathrm{CO}_{2}$ laser with $\mathrm{SF}_{6}$ as a saturable absorber, was studied. A theoretical scheme of this phenomenon was developed. Trains of laser pulses may be calculated with this model. Tests of the calculations show the dominant role of rotational relaxation in the absorber.

Introduction. - Le déclenchement passif du laser à gaz carbonique a été souvent observé, en utilisant principalement $\mathrm{SF}_{6}$ ou $\mathrm{BCl}_{3}$ comme absorbant saturable $[1,2]$. Depuis l'apparition des lasers TEA à gaz carbonique, ces études ont en partie été abandonnées. Nous avons repris des recherches sur ce phénomène, et nous présentons ici le résultat de nos observations expérimentales. D'autre part, nous proposons un modèle théorique qui permet de donner une interprétation satisfaisante du processus de création des impulsions laser.

1. Montage expérimental. - La figure 1 présente le schéma de l'expérience. La cavité a $1 \mathrm{~m}$ de longueur. Pour diminuer l'influence des dilatations, son support est constitué par deux tiges de quartz de forte section. A

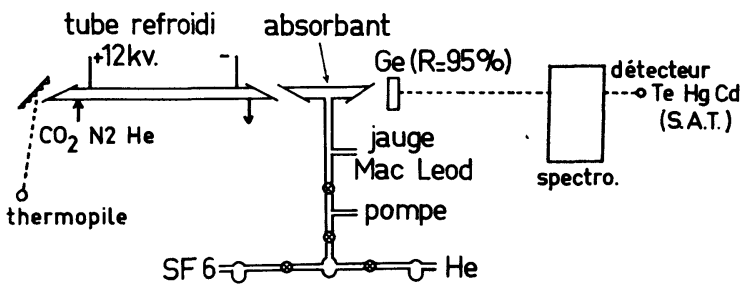

Fig. 1. - Montage expérimental.

(*) Laboratoire associé au C. N. R.S.

REVUE DE PHYSIQUE APPLIQUÉE. - T. 10, No 5, SEPTEMBRE 1975 une extrémité, un réseau en acier inoxydable de 150 traits par $\mathrm{mm}$ et de $45^{\circ}$ d'angle de miroitement sert de réflecteur et de sélecteur de fréquence. A l'autre extrémité, un miroir diélectrique transmet $5 \%$ de l'énergie à $10,6 \mu$.

L'énergie laser sort par ce miroir et est envoyée sur un spectromètre qui sert à identifier les raies laser. Le détecteur de ce spectromètre est une diode $\mathrm{TeHg}-\mathrm{TeCd}$ dont le temps de réponse est voisin de $3 \mathrm{~ns}\left({ }^{1}\right)$. Le signal électrique fourni par le détecteur est envoyé sur un oscilloscope HP183A dont le temps de réponse est inférieur à $3 \mathrm{~ns}$. De l'énergie laser est également réfléchie par l'ordre zéro du réseau. Elle est reçue sur une thermopile.

Le tube amplificateur a $70 \mathrm{~cm}$ de longueur et son diamètre interne est de $1 \mathrm{~cm}$. Le mélange $\mathrm{Co}_{2}-\mathrm{N}_{2}-\mathrm{He}$ est pompé en permanence à travers le tube. Les pressions partielles des gaz sont 0,5 torr de $\mathrm{CO}_{2}, 0,5$ torr de $\mathrm{N}_{2}$ et 1 torr de He. Le courant de décharge est de $30 \mathrm{~mA}$ sous $12 \mathrm{kV}$.

L'absorbant saturable est contenu dans une cuve de $10 \mathrm{~cm}$ de longueur. Des fenêtres en chlorure de sodium sont placées à l'incidence brewstérienne. Cette cuve est connectée à une pompe secondaire et à une installation qui permet d'admettre et de mélanger plusieurs gaz.

(1) Ce détecteur nous a été fourni par la Société Anonyme des Télécommunications. 
La pression totale dans la cuve est contrôlée à l'aide d'une jauge de MacLeod sensible de $10^{-6}$ torr à $10^{-1}$ torr.

2. Observations expérimentales. - Dans les conditions normales de fonctionnement du tube amplificateur, le régime impulsionnel apparaît pour des pressions de $\mathrm{SF}_{6}$ comprises entre $50 \mu$ et $120 \mu$ de $\mathrm{Hg}$ (Photo 1a). Comme le montre la photo $1 b$, une impul-

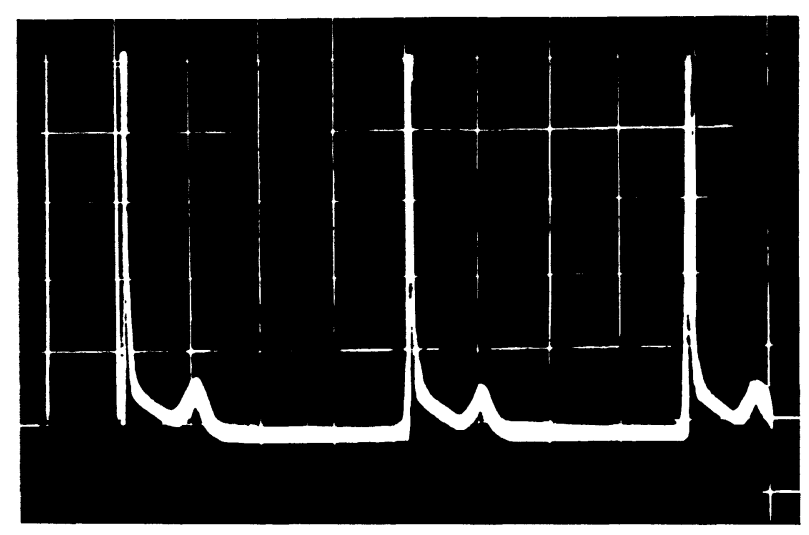

Pното 1a. - Train d'impulsions (50 $\mu \mathrm{s} /$ division).

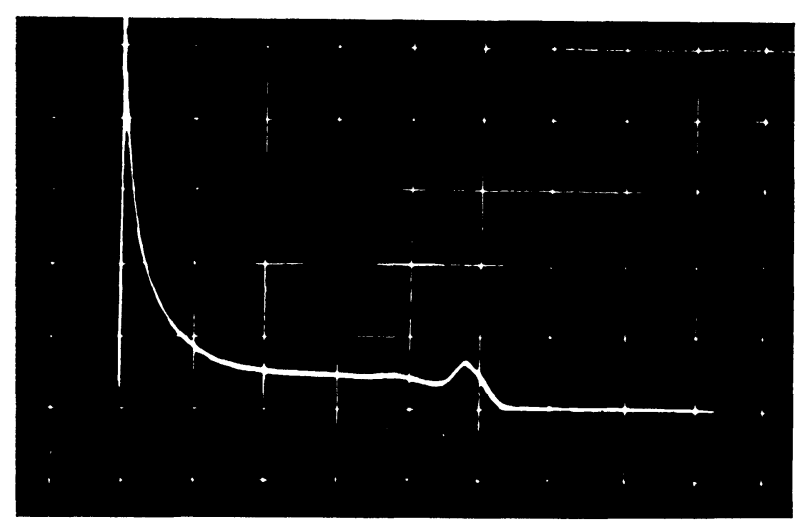

Рното $1 b$. - Une impulsion (10 $\mu$ s/division).

sion comporte un front de montée très raide. Le sommet de l'impulsion est atteint en moins de $80 \mathrm{~ns}$. La chute de l'impulsion est un peu plus lente, ce qui conduit à une largeur à mi-hauteur comprise entre 250 et 300 ns. L'impulsion est suivie d'une traîne qui est une émission presque constante dont la durée varie avec la pression de $\mathrm{SF}_{6}$. La fin de la traîne est en général marquée par une très faible impulsion. L'intervalle de temps entre deux grandes impulsions dépend de la longueur de la traîne mais n'excède jamais $600 \mu \mathrm{s}$. La forme très particulière de ces impulsions a déjà été signalée, en particulier par Burak et al. [3], mais n'a jamais fait l'objet d'aucune étude. Il faut signaler que nous avons observé une forme très semblable avec d'autres absorbants saturables, $\mathrm{BCl}_{3}$ par exemple. Nous avons entrepris de définir expérimentalement les caractères de ce phénomène.

$\mathrm{Si}$ on étudie une impulsion en faisant un balayage très rapide de l'oscillographe (Photo 2), on remarque que depuis le sommet, l'énergie laser décroît d'abord très vite, puis vers la mi-hauteur la décroissance devient plus lente. On a pu déterminer que pendant la durée de la traîne, la décroissance est exponentielle.

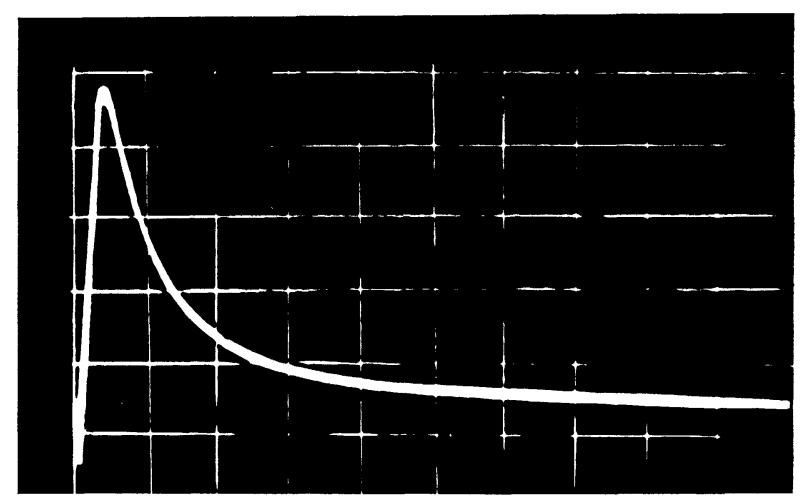

Рното 2. - Impulsion observée avec un balayage plus rapide $(0,2 \mu \mathrm{s} /$ division $)$.

Ceci implique que l'inversion de population n'ait pas été complètement détruite par le passage de l'impulsion principale, et que l'absorbant reste transparent pendant un temps suffisant. La décroissance étant très lente, les pertes (pertes fixes de la cavité plus résidu d'absorption) sont à peine supérieures au gain résiduel du tube amplificateur. Dès que l'absorbant par relaxation tend à revenir vers son état d'équilibre, les pertes l'emportent nettement et provoquent l'extinction définitive de l'émission. Il faut rappeler que l'extinction n'est pas un phénomène simple puisqu'elle est précédée par une petite remontée de l'émission laser. On peut également signaler que la traîne disparaît si l'on ajoute un gaz étranger comme de l'hélium à $\mathrm{SF}_{6}$. Ceci conduit à penser que les relaxations sont des phénomènes très importants qui peuvent modifier énormément la forme de l'impulsion laser. Ces conclusions seront vérifiées dans la quatrième partie.

Nous avons ensuite mesuré la durée de la traîne $\theta$ en faisant varier la pression $p$ de $\mathrm{SF}_{6}$. La forme de la courbe $\theta=f(p)$ nous a conduit à tester une loi expérimentale telle que :

$$
\log \theta=k(1 / p) .
$$

La figure 2 montre que cette loi est parfaitement vérifiée pour les raies $\mathbf{P}_{20}$ et $\mathbf{P}_{24}$ de la transition $v_{3}-v_{1}$. Le mécanisme du déclenchement passif se révélant très complexe dans son évolution, il n'apparaît pas possible de retrouver par la théorie la forme explicite de $\log \theta$, mais nous verrons que, par le calcul, on peut néanmoins vérifier la validité de la loi expérimentale que nous avons choisie.

Le temps entre deux impulsions est un des caractères auquel nous nous sommes intéressés. La période $T$ des impulsions dépend de la pression de l'absorbant. On observe que le temps $t=T-\theta$ qui sépare la fin de la traîne de l'impulsion suivante, varie faiblement avec 


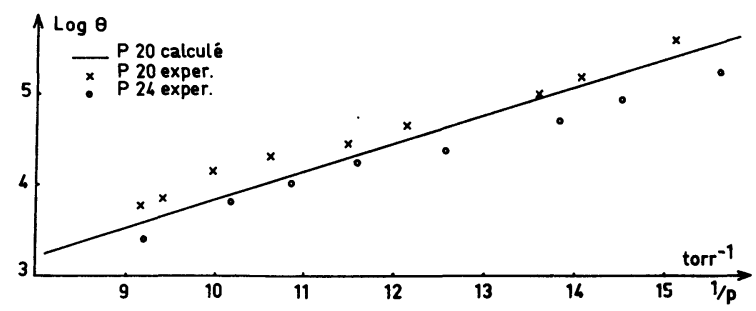

FIG. 2. - Variation de la durée de la traîne $\theta$ ( $\mu$ s) en fonction de la pression $p$ de l'absorbant saturable.

cette pression. Sur la photo $1 a$, on peut estimer cette durée à $150 \mu$ s avec une incertitude relative de $10 \%$. Cette durée est liée au temps que met le milieu amplificateur pour revenir à une inversion de population suffisante pour redéclencher l'émission laser. Ce temps est à rapprocher de la valeur $t=300 \mu$ s publiée par Javan et al. [4] dans une expérience assez différente et avec un tube amplificateur moins performant. Il faut noter que $t$ et $\theta$ sont très sensibles au coefficient de surtension de la cavité. Des vibrations peuvent affecter ces valeurs. Toutes nos mesures ont donc été faites en isolant au mieux la cavité des vibrations extérieures.

3. Calcul théorique de la forme des impulsions laser. - 3.1 ÉTABLISSEMENT DES ÉQUATIONS D'ÉVOLUTION. La reconstitution des impulsions émises par un laser contenant un absorbant saturable a été entreprise pour la première fois par Szabo et Stein [5] et Hofelich-Abate et al. [6], pour expliquer la génération d'onde géante par un laser à rubis contenant un absorbant saturable. Le mécanisme était décrit par trois équations différentielles qui fournissent l'évolution de la densité de photons dans la cavité, l'évolution de l'inversion de population dans le milieu amplificateur, et l'évolution des populations dans l'absorbant.

Ce schéma se révèle trop simpliste pour être appliqué directement à un milieu moléculaire. Une excellente approche de la résolution a été donnée par Burak et al. [3]. Son calcul repose sur un modèle raisonnable qui décrit tous les échanges d'énergie à l'intérieur des deux milieux moléculaires. Rappelons ici le modèle proposé schématisé sur la figure 3 . Aussi bien pour le milieu amplificateur que pour le milieu absorbant, on considère les deux niveaux rovibrationnels $J_{1}$ et $J_{2}$ qui sont liés par le phénomène radiatif. Nous appellerons :

$N_{\mathrm{J}_{1}^{\prime}}$ et $N_{\mathrm{J}_{2}^{\prime}}$ les populations des niveaux $\mathrm{J}_{1}^{\prime}, \mathrm{J}_{2}^{\prime}$ de l'absorbant et $M_{\mathrm{J}_{1}}$ et $M_{\mathrm{J}_{2}}$ les populations des niveaux $\mathbf{J}_{1}, \mathbf{J}_{2}$ de l'amplificateur.

Toutes les variables relatives à l'absorbant seront indicées $N$, celles relatives à l'amplificateur, $M$.

Les niveaux rotationnels $\mathbf{J}_{1}, \mathbf{J}_{2}, \mathbf{J}_{1}^{\prime}$ et $\mathbf{J}_{2}^{\prime}$ sont entourés par d'autres niveaux rotationnels du même niveau vibrationnel $\mathrm{v}$ avec lesquels il peut $\mathrm{y}$ avoir échange de molécules par relaxation rotationnelle. La base du calcul consiste à admettre que ces niveaux rovibrationnels autres que $J_{1}, J_{2}, J_{1}^{\prime}$ et $J_{2}^{\prime}$ forment un ensemble

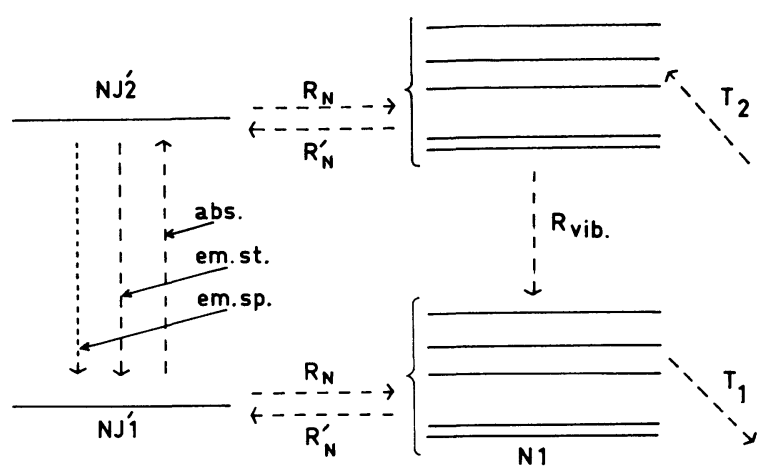

(a)

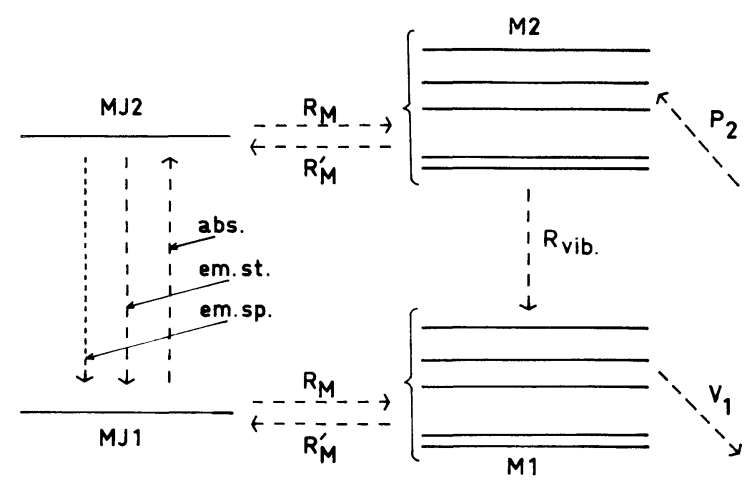

(b)

FIG. 3. - Représentation schématique d'un modèle à 4 niveaux. a) Dans l'amplificateur. b) Dans l'absorbant saturable.

non dissociable. Nous appellerons $N_{1}, N_{2}, M_{1}, M_{2}$ la population totale de ces niveaux rovibrationnels respectivement dans l'absorbant et dans l'amplificateur. La relaxation entre les niveaux $J_{1 \text { ou } 2}^{\prime}$ et $N_{1 \text { ou } 2}$ dans l'absorbant ( $\mathrm{J}_{1 \text { ou } 2}$ et $M_{1 \text { ou } 2}$ dans l'amplificateur) sera décrite par une constante de relaxation que nous nommerons $R_{N}\left(R_{M}\right)$ : Burak et al. ont montré que cette constante doit être choisie un peu inférieure à la constante de relaxation rotationnelle $k_{\mathrm{i}-\mathrm{J}}$.

A partir de ce modèle, on voit que $N_{\mathrm{J}_{1}^{\prime}}$ et $N_{\mathrm{J}_{2}^{\prime}}$ vont évoluer sous l'action de plusieurs phénomènes :

- Le phénomène radiatif correspondant à la transition $\mathbf{J}_{2}^{\prime} \rightarrow \mathbf{J}_{1}^{\prime}$ est amplifié ou réabsorbé par les deux milieux moléculaires. C'est le phénomène dominant dans la création des impulsions laser.

- La relaxation rotationnelle $N \rightleftarrows N_{\mathrm{J}^{\prime}}$ et $M \rightleftarrows M_{\mathrm{J}}$, qui tend à créer dans les états vibrationnels un pseudoéquilibre dans leurs populations rotationnelles.

- Le pompage qui tend à ramener la population $M_{2}$, vers son état de pseudo-équilibre décrit par une température vibrationnelle très élevée.

- La relaxation vibrationnelle qui tend à ramener $M_{1}, N_{1}, N_{2}$ vers leur état d'équilibre.

Nous avons établi les équations qui déterminent l'évolution de la densité de photons et des populations. Elles forment un système de neuf équations différentielles couplées (Tableau I) que nous analysons cidessous. 


\section{TABLEAU I}

$$
\begin{aligned}
\frac{\mathrm{d} \varphi}{\mathrm{d} t} & =\left\{(1-\Gamma) \exp \left[A_{M} l\left(\frac{M_{\mathrm{J}_{2}}}{g_{2}}-\frac{M_{\mathrm{J}_{1}}}{g_{1}}\right)-A_{N} d\left(\frac{N_{\mathrm{J}_{1}^{\prime}}}{g_{1}^{\prime}}-\frac{N_{\mathrm{J}_{2}^{\prime}}}{g_{2}^{\prime}}\right)\right]-1\right\} \frac{c}{L} \varphi+S_{M} M_{\mathrm{J}_{2}}+S_{N} N_{\mathrm{J}_{2}^{\prime}} \\
\frac{\mathrm{d} M_{\mathrm{J}_{1}}}{\mathrm{~d} t} & =A_{M}\left(\frac{M_{\mathrm{J}_{2}}}{g_{2}}-\frac{M_{\mathrm{J}_{1}}}{g_{1}}\right) c \varphi-R_{M} M_{\mathrm{J}_{1}}+R_{M_{1}}^{\prime} \cdot M_{1} \\
\frac{\mathrm{d} M_{\mathrm{J}_{2}}}{\mathrm{~d} t} & =-A_{M}\left(\frac{M_{\mathrm{J}_{2}}}{g_{2}}-\frac{M_{\mathrm{J}_{1}}}{g_{1}}\right) c \varphi-R_{M} M_{\mathrm{J}_{1}}+R_{M_{2}}^{\prime} \cdot M_{2} \\
\frac{\mathrm{d} M_{1}}{\mathrm{~d} t} & =R_{M} M_{\mathrm{J}_{1}}-R_{M_{1}}^{\prime} \cdot M_{1}-V_{1}\left(M_{1}-M_{1}^{*}\right) \\
\frac{\mathrm{d} M_{2}}{\mathrm{~d} t} & =R_{M} M_{\mathrm{J}_{2}}-R_{M_{2}}^{\prime} \cdot M_{2}-P_{2}\left(M_{2}-M_{2}^{*}\right) \\
\frac{\mathrm{d} N_{\mathrm{J}_{1}^{\prime}}}{\mathrm{d} t} & =-A_{N}\left(\frac{N_{\mathrm{J}_{1}^{\prime}}^{\prime}}{g_{1}^{\prime}}-\frac{N_{\mathrm{J}_{2}^{\prime}}}{g_{2}^{\prime}}\right) c \varphi-R_{N^{\prime}} \cdot N_{\mathrm{J}_{1}^{\prime}}+R_{N_{1}}^{\prime} \cdot N_{1} \\
\frac{\mathrm{d} N_{\mathrm{J}_{2}^{\prime}}}{\mathrm{d} t} & =A_{N}\left(\frac{N_{\mathrm{J}_{1}^{\prime}}}{g_{1}^{\prime}}-\frac{N_{\mathrm{J}_{2}^{\prime}}^{\prime}}{g_{2}^{\prime}}\right) c \varphi-R_{N} N_{\mathrm{J}_{2}^{\prime}}+R_{N_{2}}^{\prime} \cdot N_{2} \\
\frac{\mathrm{d} N_{1}}{\mathrm{~d} t} & =R_{N} N_{\mathrm{J}_{1}^{\prime}}-R_{N_{1}}^{\prime} \cdot N_{1}-T_{1}\left(N_{1}-N_{1}^{0}\right)+R_{\mathrm{vib}} \cdot N_{2} \\
\frac{\mathrm{d} N_{2}}{\mathrm{~d} t} & =R_{N} N_{\mathrm{J}_{2}^{\prime}}-R_{N_{2}}^{\prime} \cdot N_{2}-T_{2}\left(N_{2}-N_{2}^{0}\right)-R_{\mathrm{vib}} \cdot N_{2} .
\end{aligned}
$$

a) Nombre de photons par unité de volume d'un mode à l'intérieur de la cavité :

Les conditions particulières de fonctionnement d'un laser à gaz carbonique (gain et pertes par couplage très élevés) nous imposent d'écrire très rigoureusement cette équation.

L'onde dont la densité de photons est $\varphi$ se propage dans la cavité de longueur $L$. Elle subit une absorption dans la cuve de longueur $d$ et sa densité devient $\varphi \mathrm{e}^{-\alpha_{N} d}$. Elle est ensuite amplifiée dans le tube luminescent de longueur $l$ et sa densité atteint $\varphi \mathrm{e}^{\alpha_{M} l-\alpha_{N} d}$, $\alpha_{M}$ et $\alpha_{N}$ sont positifs. Sur le miroir l'onde subit des pertes de pourcentage $\Gamma$. La fraction réfléchie est donc $\varphi(1-\Gamma) \mathrm{e}^{\alpha_{M} l-\alpha_{N} d}$. Pendant le temps $\mathrm{d} t=L / c$, que l'onde met à traverser la cavité, elle subit une variation de sa densité de photons qui peut s'exprimer par:

$$
\frac{\mathrm{d} \varphi}{\mathrm{d} t}=\left[(1-\Gamma) \mathrm{e}^{\alpha_{M} l-\alpha_{N} d}-1\right] \frac{c}{L} \varphi .
$$

Nous pouvons expliciter $\alpha_{M}$ ou $\alpha_{N}$ au sommet de raies élargies par effet Doppler. Des corrections seraient nécessaires, en particulier dans l'amplificateur, pour tenir compte de l'élargissement mixte qui se traduit par la forme de Voigt. Nous pouvons écrire pour l'amplificateur :

$$
\alpha_{M}=\sqrt{\frac{\log 2}{\Pi}} \frac{1}{\gamma_{\mathrm{D}}} B_{21} \mathrm{~g}_{1}\left[\frac{M_{\mathrm{J}_{2}}}{g_{2}}-\frac{M_{\mathrm{J}_{1}}}{g_{1}}\right] \frac{h v}{c}
$$

où $B_{21}$ est le coefficient d'Einstein d'émission induite correspondant à la transition $2 \rightarrow 1, g_{1}=2 \mathrm{~J}_{1}+1$, $g_{2}=2 \mathrm{~J}_{2}+1$, soit sous une forme plus développée :

$$
\begin{aligned}
\alpha_{M}=\sqrt{\frac{\log 2}{\Pi}} \frac{1}{\gamma_{\mathrm{D}}} \frac{8 \Pi^{3} \sigma}{3 h c}|m| \mid & \left.R_{\mathrm{v}}^{\mathrm{v}^{\prime}}(m)\right|^{2} \times \\
& \times\left(\frac{M_{\mathrm{J}_{2}}}{g_{2}}-\frac{M_{\mathrm{J}_{1}}}{g_{1}}\right)
\end{aligned}
$$

où $\gamma_{\mathrm{D}}$ est la demi-largeur Doppler de la raie, $\sigma$ son nombre d'onde, $v$ fréquence, $|m|$ est la probabilité de transition rotationnelle et vaut $\mathrm{J}$ pour une raie $\mathbf{P}$ et $(\mathrm{J}+1)$ pour une raie $R,\left|\mathbf{R}_{\mathrm{v}}^{\mathrm{v}^{\prime}}(m)\right|^{2}$ est la probabilité de transition vibrationnelle. Pour simplifier l'écriture nous poserons :

$$
A_{M}=\sqrt{\frac{\log 2}{\Pi}} \frac{1}{\gamma_{\mathrm{D}}} \frac{8 \Pi^{3} \sigma}{3 h c}|m|\left|R_{\mathrm{v}}^{\mathrm{v}^{\prime}}(m)\right|^{2} .
$$

Dans l'éq. (1) nous devons ajouter deux termes d'émission spontanée $S_{N}$ et $S_{M}$ provenant de la cuve et du tube amplificateur. Leur contribution est excessivement faible. Cette émission joue un rôle néanmoins capital, puisqu'elle crée dans la cavité les quelques photons nécessaires à faire démarrer l'émission laser. L'équation complète est présentée dans le tableau I. Il faut remarquer qu'elle prend la forme adoptée par d'autres auteurs $[3,5,6]$, quand l'absorption, l'amplification et les pertes sont supposées négligeables. Soit :

$$
\begin{aligned}
& \frac{\mathrm{d} \varphi}{\mathrm{d} t}=\left\{A_{M} l\left(\frac{M_{\mathrm{J}_{2}}}{g_{2}}-\frac{M_{\mathrm{J}_{1}}}{g_{1}}\right)-\right. \\
& \left.-A_{N} d\left(\frac{N_{\mathrm{J}_{1}^{\prime}}}{g_{1}^{\prime}}-\frac{N_{\mathrm{J}_{2}^{\prime}}}{g_{2}^{\prime}}\right)-\Gamma\right\} \frac{c}{L} \varphi+S_{M} M_{\mathrm{J}_{2}}+S_{N} N_{\mathrm{J}_{2}^{\prime}}
\end{aligned}
$$


b) Etablissement des équations d'évolution dans l'amplificateur. - Suivant le schéma adopté, nous devons introduire quatre équations qui régissent respectivement l'évolution des populations $M_{\mathrm{J}_{1}}, M_{\mathrm{J}_{2}}, M_{1}, M_{2}$.

Pour $M_{\mathrm{J}_{1}}$ et $M_{\mathrm{J}_{2}}$, il faut retenir d'abord le rôle de l'absorption et de l'émission induite sous l'effet de la densité de photons $\varphi$ soit :

$$
\frac{\mathrm{d} M_{\mathrm{J}_{1}}}{\mathrm{~d} t}=-B_{12}\left|M_{\mathrm{J}_{1}}-\frac{g_{1}}{g_{2}} M_{\mathbf{J}_{2}}\right| \rho
$$

avec

$$
\rho=h c \sigma \varphi
$$

soit :

$$
\frac{\mathrm{d} M_{\mathrm{J}_{1}}}{\mathrm{~d} t}=A_{M}\left|\frac{M_{\mathrm{J}_{2}}}{g_{2}}-\frac{M_{\mathrm{J}_{1}}}{g_{1}}\right| c \varphi
$$

et :

$$
\frac{\mathrm{d} M_{\mathrm{J}_{2}}}{\mathrm{~d} t}=-A_{M}\left|\frac{M_{\mathrm{J}_{2}}}{g_{2}}-\frac{M_{\mathrm{J}_{1}}}{g_{1}}\right| c \varphi .
$$

Nous devons ensuite tenir compte de la relaxation rotationnelle qui tend à rééquilibrer le niveau rovibrationnel $J_{1}$ ou $J_{2}$ avec les autres niveaux rotationnels du même niveau vibrationnel. Nous écrirons :

$$
\begin{aligned}
& \frac{\mathrm{d} M_{\mathrm{J}_{1}}}{\mathrm{~d} t}=-R_{M}^{1}\left(M_{\mathrm{J}_{1}}-M_{\mathrm{J}_{1}}^{\mathrm{eqR}}\right) \\
& \frac{\mathrm{d} M_{\mathrm{J}_{2}}}{\mathrm{~d} t}=-R_{M}^{2}\left(M_{\mathrm{J}_{2}}-M_{\mathrm{J}_{2}}^{\mathrm{eqR}}\right) .
\end{aligned}
$$

$M_{\mathrm{J}_{1}}^{\text {eqR }}$ et $M_{\mathrm{J}_{2}}^{\text {eqR }}$, populations de $\mathrm{J}_{1}$ et $\mathrm{J}_{2}$ quand l'équilibre rotationnel est atteint, peuvent être exprimés en fonction de $M_{1}$ et $M_{2}$

$$
\begin{aligned}
& M_{\mathrm{J}_{1}}^{\mathrm{eqR}}=\beta_{1} M_{1}^{\mathrm{total}}=\beta_{1}\left(M_{1}^{\mathrm{eq}}+M_{\mathrm{J}_{1}}^{\mathrm{eq}}\right)=\frac{\beta_{1}}{1-\beta_{1}} M_{1} \\
& M_{\mathrm{J}_{2}}^{\mathrm{eqR}}=\beta_{2} M_{2}^{\mathrm{total}}=\beta_{2}\left(M_{2}^{\mathrm{eq}}+M_{\mathrm{J}_{2}}^{\mathrm{eq}}\right)=\frac{\beta_{2}}{1-\beta_{2}} M_{2} .
\end{aligned}
$$

Nous admettrons que les taux de relaxation rotationnels $R_{M}^{1}$ et $R_{M}^{2}$, pour les niveaux inférieur et supérieur, sont égaux : $R_{M}^{1}=R_{M}^{2}=R_{M}$. Nous poserons en outre :

$$
R_{M}^{\prime}=\frac{\beta}{1-\beta} R_{M}
$$

Il vient finalement :

$$
\begin{aligned}
& \frac{\mathrm{d} M_{\mathrm{J}_{1}}}{\mathrm{~d} t}=-R_{M} M_{\mathrm{J}_{1}}+R_{M_{1}}^{\prime} M_{1} \\
& \frac{\mathrm{d} M_{\mathrm{J}_{2}}}{\mathrm{~d} t}=-R_{M} M_{\mathrm{J}_{2}}+R_{M_{2}}^{\prime} M_{2} .
\end{aligned}
$$

On peut également tenir compte du terme d'émission spontanée : $+S_{M} M_{\mathrm{J}_{2}}$ dans [4] et $-S_{M} M_{\mathrm{J}_{2}}$ dans [5].

Ce terme a peu d'influence et dans la pratique il peut être négligé.
Le tableau I fournit l'expression complète de ces deux équations.

Pour $M_{1}$ et $M_{2}$, on doit également retenir le rôle de la relaxation rotationnelle, soit :

$$
\begin{aligned}
& \frac{\mathrm{d} M_{1}}{\mathrm{~d} t}=R_{M} M_{\mathrm{J}_{1}}-R_{M_{1}}^{\prime} M_{1} \\
& \frac{\mathrm{d} M_{2}}{\mathrm{~d} t}=R_{M} M_{\mathrm{J}_{2}}-R_{M_{2}}^{\prime} M_{2}
\end{aligned}
$$

en utilisant les notations précédentes.

Pour l'état 2, qui est le niveau vibrationnel $v_{3}$, un terme que nous appelons terme de pompage $P_{2}$ tend à amener la population $M_{2}$ vers son état de pseudoéquilibre $M_{2}^{*}$, définies par une température vibrationnelle $T_{\mathrm{v}}[12]$

$$
\frac{\mathrm{d} M_{2}}{\mathrm{~d} t}=-P_{2}\left(M_{2}-M_{2}^{*}\right) .
$$

De même pour l'état 1, quand il se trouve surpeuplé, on doit introduire un terme de relaxation qui le ramène à sa valeur stationnaire, $M_{1}^{*}$ [12] soit :

$$
\frac{\mathrm{d} M_{1}}{\mathrm{~d} t}=-V_{1}\left(M_{1}-M_{1}^{*}\right) \text {. }
$$

Nous disposons de deux équations supplémentaires qui figurent dans le tableau I.

c) Etablissement des équations d'évolution des populations dans l'absorbant. - Les équations sont du même type que dans l'amplificateur. Il n'y a pas dans l'absorbant de terme de pompage, par contre, il est légitime d'introduire un terme que nous désignons par $T_{1}$ ou $T_{2}$ et qui décrit le rééquilibrage thermodynamique. Dans l'écriture, $N_{1}^{0}$ et $N_{2}^{0}$, sont les populations des niveaux 1 et 2 à la température $T$ et à l'équilibre. D'autre part étant donnée la forte probabilité de transition, un terme de relaxation par fluorescence doit être pris en compte. Il est décrit par un coefficient noté $R_{\mathrm{vib}}$ Les quatre équations relatives à l'absorbant sont fournies par le tableau I. En définitive, nous disposons de neuf équations différentielles à neuf inconnues. La résolution de ce système va nous conduire à rendre compte de façon satisfaisante du phénomène de déclenchement passif.

3.2 RÉSOLUTION DU SYSTÈME. - La résolution du système a été rendue possible par l'utilisation de l'ordinateur UNIVAC 1110 de l'Université de Paris XI. L'adaptation du programme de résolution par la méthode de Runge-Kutta à notre problème nous permet de retracer la forme des impulsions pour des intervalles de temps de $3 \mathrm{~ns}$, correspondant à chaque passage de l'onde dans la cavité. La première application que nous ayons faite a été adaptée à $\mathrm{SF}_{6}$.

Les paramètres figurant dans les équations ont été fixés aux valeurs les plus fiables publiées dans la littérature. Dans l'amplificateur, nous avons retenu les valeurs suivantes: 
- Pour le terme $A_{M}$, nous avons choisi les résultats publiés en référence [7]. Pour la raie $\mathbf{P}_{20}$ de la transition $v_{3}-v_{1}$ nous avons retenu la valeur :

$$
A_{M}=6,2 \times 10^{-15} \mathrm{~cm}^{-1} \text { molécule }^{-1} \text {. }
$$

- Le coefficient de pertes $\Gamma$ a été estimé dans notre montage à $\Gamma=0,20$.

- Le coefficient $\beta$ est défini par la loi de Boltzman à la température de rotation $T_{\mathrm{R}}$. Il a été calculé en utilisant les tables de Gray [8], ainsi que les constantes rotationnelles connues de $\mathrm{CO}_{2}$.

- Le terme $R_{M}$ de relaxation rotationnelle, est un paramètre qui a fait l'objet de nombreuses mesures. Dans les conditions de pression des gaz utilisés dans le tube luminescent, nous avions le choix entre les valeurs de : $10^{7} \mathrm{~s}^{-1}$ déterminée par Chéo et Abrams [9], $1,5 \times 10^{7}$ publiée par Lécuyer [10], et $1,8 \times 10^{7}$ selon Jacobs [11]. Cette dernière valeur nous a donné entière satisfaction comme on le verra plus loin.

- Les valeurs de $V_{1}$ et $P_{2}$ jouent un rôle très important dans le phénomène et nous discuterons leur choix dans le dernier paragraphe.

- Les valeurs stationnaires des populations dans le plasma, $M_{1}^{*}$ et $M_{2}^{*}$ ont été fixées à celles déterminées par D. Bailly [12].

Dans l'absorbant, les paramètres sont beaucoup moins bien déterminés.

- Par mesure directe de l'absorption de la raie $\mathbf{P}_{20}$ du laser, nous avons déterminé le coefficient d'absorption $\alpha_{\text {exp }}$. En se reportant aux équations du tableau I nous pouvons définir :

$$
A_{N}=\frac{\alpha_{\mathrm{exp}}}{\frac{N_{\mathrm{J}_{1}^{\prime}}}{g_{1}^{\prime}}-\frac{N_{\mathrm{J}_{2}^{\prime}}}{g_{2}^{\prime}}} .
$$

Il ne peut être calculé si l'on ne connaît pas les niveaux d'énergie de $\mathrm{SF}_{6}$. En effet :

$$
\frac{N_{\mathrm{J}_{1}^{\prime}}^{\prime}}{g_{1}^{\prime}}=N \frac{\mathrm{e}^{-h c \sigma_{1} / k T}}{Z_{\mathrm{v}} Z_{\mathrm{R}}} \mathrm{e}^{-h c B_{1}} \frac{J(J+1)}{k T}=\frac{\beta_{1} N}{Z_{\mathrm{v}}} \mathrm{e}^{-h c \sigma_{1} / k T} .
$$

Des mesures conduisent à une valeur

$$
\beta_{1}=\beta_{2} \approx 1 / 300 \text { [13], [14] }
$$

Il nous faut encore connaître la fonction de partition vibrationnelle $Z_{\mathrm{v}}$ et $\sigma_{1}$. La figure 4 présente les valeurs connues des niveaux d'énergie vibrationnels $S_{6}$. Nous en déduisons :

$$
Z_{\mathrm{v}}=3,0 \text {. }
$$

Certains auteurs ont été amenés à penser que la transition active pourrait être $v_{3}+v_{6}-v_{6}$. Nous n'avons pas retenu cette hypothèse et nous avons admis que le niveau de départ de la transition qui absorbe est le niveau fondamental de la molécule. Il en découle une valeur de $A_{N}=7,9 \times 10^{-13} \mathrm{~cm}^{-1} / \mathrm{mol}$. Le terme de relaxation vibrationnelle a été choisi à la valeur [13] :

$$
R^{\mathrm{vib}}=2,5 \times 10^{4} \mathrm{~s}^{-1} .
$$

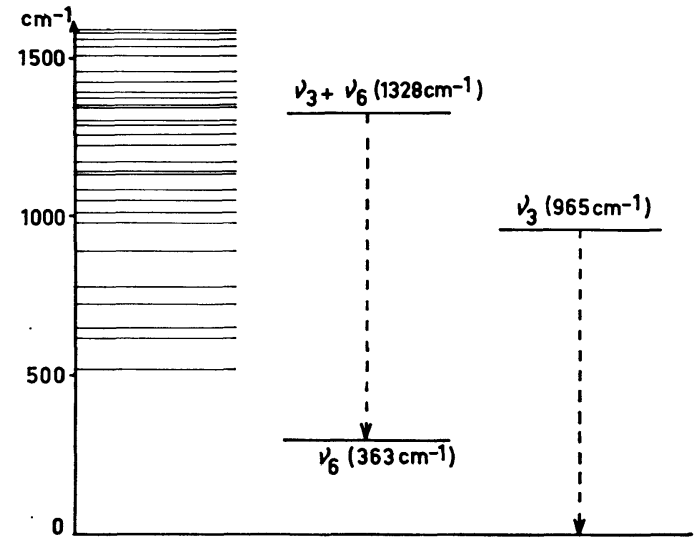

FIG. 4. - Représentation de quelques niveaux d'énergie vibrationnels de la molécule de $\mathrm{SF}_{6}$.

La valeur de $R_{N}$ a été déterminée par des mesures de relaxation rotationnelle de $\mathrm{SF}_{6}$ par He [15]. La valeur de $R_{N}=10^{7} \mathrm{~s}^{-1}$ nous a permis de rendre compte de la forme exacte des impulsions.

Avec cet échantillonnage de valeurs, nous avons pu recalculer les impulsions présentées sur la figure 5 . Elle représente comme nous l'espérions des impulsions de 300 ns de largeur chaque impulsion étant suivie d'une traîne de très longue durée. Ce profil peut être comparé avec l'une des photos précédentes. Nous allons présenter les tests effectués sur notre calcul et discuter les hypothèses que nous avons faites.

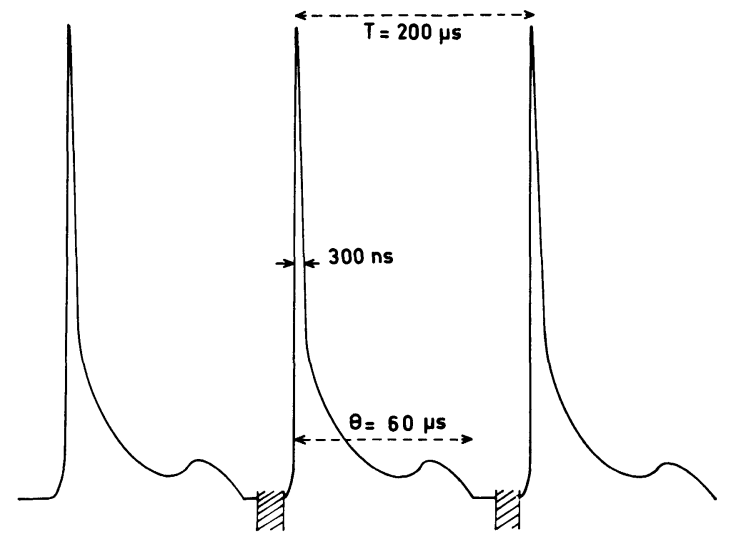

Fig. 5. - Reconstitution par ordinateur d'un train d'impulsions.

4. Résultats des calculs. - Etant donnée la longueur des temps de calcul, il n'est pas possible de tester tous les paramètres qui apparaissent dans nos équations. En particulier, nous avons admis comme connues les valeurs de $A_{M}$ dans l'amplificateur. Pour l'absorbant, nous supposons également connus $A_{N}$ et $\beta_{N}$. Nous nous sommes attachés à l'étude du terme de pompage $P_{2}$, du terme de relaxation vibrationnelle $V_{1}$ et de relaxation rotationnelle $R_{M}$ dans le plasma. Dans l'absorbant, nous avons testé le rôle du terme de relaxation rotationnelle $R_{N}$ et de la pression. 
4.1 RôLES DE $V_{1}$ ET $P_{2}$. $-P_{2}$ est lié à l'inverse du temps d'excitation du niveau $v_{3}$. Les collisions électroniques sont dominantes. Avec la valeur de $E / N$ choisie par Nhigan [16], on trouve pour 1 torr de $\mathrm{CO}_{2}$, une constante de temps de $200 \mu \mathrm{s}$, soit $P_{2}^{\prime}=5 \times 10^{3} \mathrm{~s}^{-1}$.

Il faut également tenir compte de l'excitation du niveau $v_{3}$ par les transferts d'énergie $\mathrm{V}-\mathrm{V}$ dus à l'azote activé. La constante de temps de ce processus est voisine de la précédente [17]. Ce sont donc deux processus extrêmement lents. Il existe d'autres processus qui sont moins efficaces mais beaucoup plus rapides. Par exemple Sharma [17] propose le mécanisme suivant :

$$
\mathrm{CO}_{2}\left(2 v_{3}\right)+\mathrm{CO}_{2}(0) \rightarrow 2 \mathrm{CO}_{2}\left(v_{3}\right) \text {. }
$$

Il estime la constante de temps de ce processus à $200 \mathrm{~ns}$, soit $P_{2}^{\prime \prime}=5 \times 10^{6} \mathrm{~s}^{-1}$ torr $^{-1}$. Dans le même ordre d'idée, Burak [18] a mesuré la constante de temps de l'équation collisionnelle suivante :

$$
\mathrm{CO}_{2}\left(v_{3}+v_{2}\right)+\mathrm{CO}_{2}(0) \rightarrow \mathrm{CO}_{2}\left(v_{3}\right)+\mathrm{CO}_{2}\left(v_{2}\right) .
$$

Il a obtenu la valeur $P_{2}$ très voisine de l'estimation de Sharma. Ces processus sont certainement peu actifs étant donné le nombre faible de molécules dans $2 v_{3}$ et $v_{3}+v_{2}$. Mais étant très rapides, ils pourraient certainement modifier quelque peu la forme de l'impulsion. Pour introduire dans notre méthode de calcul ces nouveaux mécanismes, il faudrait ajouter à notre système une équation sur l'évolution de la population de $2 v_{3}$ et une sur celle de $v_{3}+v_{2}$. Ce travail est en cours. Jusqu'ici nous admettons que seuls les processus lents déterminent l'excitation de $\mathrm{CO}_{2}$ et dans les conditions de fonctionnement de notre tube, le temps $T$ entre impulsions nous a conduit à choisir :

$$
P_{2}=3 \times 10^{+4} \mathrm{~s}^{-1} \text {. }
$$

Pour $V_{1}$, les processus sont au moins aussi complexes. Ici il s'agit de la relaxation du niveau $v_{1}$. En général, on admet quelle s'opère par transferts d'énergie vers le niveau $v_{2}$. La constante de temps de ce mécanisme est voisine de $1 \mathrm{~ms}$ pour 1 torr de $\mathrm{CO}_{2}$ [19]. Mais dans un régime impulsionnel, il peut arriver que $v_{1}$ se trouve très surpeuplé par rapport au niveau voisin $2 v_{2}$. Or ces deux niveaux sont couplés par une forte résonance de Fermi qui rend le passage de l'un à l'autre très probable sous l'effet d'une collision. Un schéma de relaxation vraisemblable consiste donc à admettre que si une impulsion laser se déclenche sur la transition $v_{3}-v_{1}, v_{1}$ se trouve surpeuplé par rapport à $2 v_{2}$. Très rapidement les deux niveaux se remettent en équilibre entre eux. Donc $v_{1}$ et $2 v_{2}$ sont tous deux surpeuplés et la relaxation vers $v_{2}$ les remet lentement en équilibre thermodynamique. Un tel processus nécessite également l'écriture de deux nouvelles équations d'évolution, une pour $v_{2}$ et une pour $2 v_{2}$. Ce système sera utilisé pour interpréter le Q-switch passif produit par $\mathrm{CO}_{2}$ lui-même. Ici nous utilisons comme seul processus de relaxation celui vers $2 v_{2}$. Nous avons choisi pour $V_{1}$ l'expression expérimentale fournie par Stark [20], en fonction des constituants du plasma :

$V_{1}=1,4 \times 10^{5} \times$

$$
\times\left(P_{\mathrm{CO}_{2}}+0,4 P_{\mathrm{N}_{2}}+0,05 P_{\mathrm{He}}\right)=9 \times 10^{4} \mathrm{~s}^{-1}
$$

pour les conditions de pression de notre tube.

Les valeurs de $V_{1}$ et $P_{2}$ choisies permettent de rendre compte des impulsions régulièrement espacées. Le temps $t=T-\theta$ mesuré sur la reconstitution est de $140 \mu \mathrm{s}$, en accord avec l'expérience (Fig. 5).

Nous avons pu constater que pour un coefficient de pertes donné, $t$ est presque exclusivement déterminé par le choix de $V_{1}$ et $P_{2}$. Le jeu de valeurs choisies est le seul qui donne une forme très réaliste de l'impulsion et qui fournit une valeur satisfaisante de $t$. Nous pensons donc avoir, sur ce point approché la réalité d'assez près.

4.2 RôLE DE LA RELAXATION ROTATIONNELLE DANS $\mathrm{CO}_{2}$. - Bien que nous disposions d'excellentes et nombreuses mesures de ce paramètre, nous avons cherché à définir son rôle dans la forme de l'impulsion.

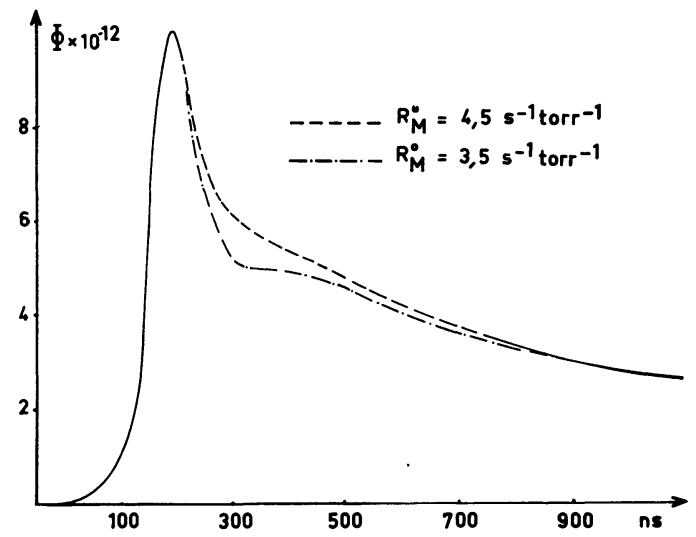

FIG. 6. - Influence du paramètre de relaxation rotationnelle de $\mathrm{CO}_{2}$ sur la forme de l'impulsion.

La figure 6/ présente la partie centrale de la reconstitution. Seul le paramètre $R_{M}$ a varié d'une courbe à l'autre. Nous ne présentons, pour la clarté du dessin, que les courbes correspondant aux valeurs extrêmes de $\boldsymbol{R}_{\boldsymbol{M}}$ parmi celles que nous avons testées. On constate que si $R_{M}$ est trop faible, il se produit un creux entre l'impulsion principale et le début de la traîne. Par contre pour des valeurs de $R_{M}$ plus élevées, la décroissance est monotone. On constate que la traîne commence d'autant plus tôt que $R_{M}$ est grand. L'influence de terme $R_{M}$ est donc très importante, bien qu'il ne modifie pas de façon fondamentale la forme de l'impulsion, tout au moins dans le domaine de variation où nous l'avons étudiée. Il faut signaler que l'on peut observer expérimentalement les formes présentées sur cette figure en faisant varier les pressions des constituants de la décharge (Photo 3). 


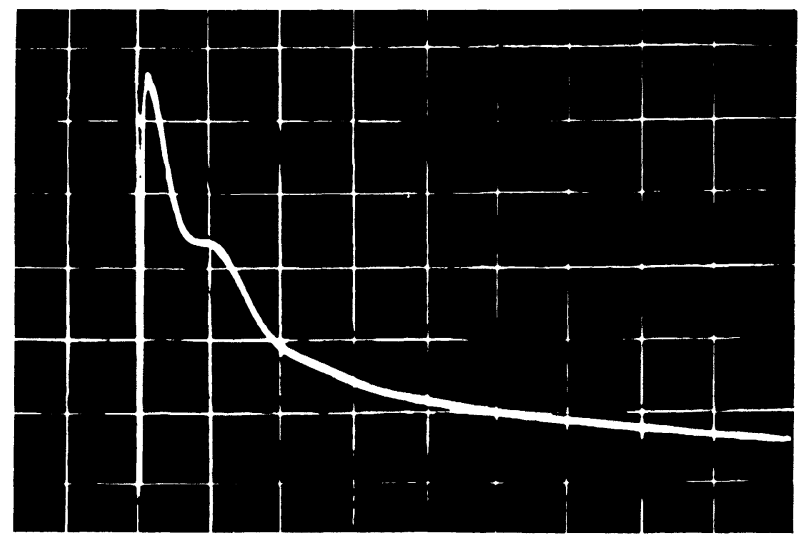

Рното 3. - Impulsion observée avec une proportion d'azote plus faible dans la composition du mélange amplificateur.

4. 3 RôLE DE LA PRESSION D'ABSORBANT SATURABLE. La pression d'absorbant saturable joue un rôle double : elle change le terme de relaxation rotationnelle :

$$
R_{N}=R_{N}^{0} p
$$

et elle change les populations $N_{1}, N_{2}, N_{\mathrm{J}_{1}^{\prime}}, N_{\mathrm{J}_{2}^{\prime}}$. Nous constatons que $\log \theta$ est, comme dans l'expérience, inversement proportionnel à $p$ (Fig. 2). En ajustant la valeur de $R_{N}^{0}$, on peut espérer obtenir une droite dont la pente soit égale à celle de la droite expérimentale. La mesure expérimentale de $\log \theta$ en fonction de la pression peut apparaître comme une méthode d'estimation de la valeur de $R_{N}$ dans le cas de molécules aussi complexes que $\mathrm{SF}_{6}$ ou $\mathrm{BCl}_{3}$ pour lesquelles on ne dispose pas de mesures expérimentales des largeurs des raies de vibration-rotation. D'autre part, nous avons fait un calcul dans lequel nous avons maintenu la pression constante, et nous avons fait varier le coefficient $R_{N}^{0}$ seulement. Si nous portons $\log \theta$ en fonction de $R_{N}^{0}$, nous obtenons la même loi de variation que dans le test précédent. Nous devons donc conclure que le paramètre qui détermine la forme et la durée de la traîne est uniquement le terme de relaxation rotationnelle.

4.4 ÉVOlUtion DES POPULATIONS. - Les évolutions au cours du temps de $N_{\mathrm{J}_{1}^{\prime}}, N_{\mathrm{J}_{2}^{\prime}}, M_{\mathrm{J}_{1}}$ et $M_{\mathrm{J}_{2}}$ sont présentées sur les figures 7 et 8 ; les figures $7 a$ et $7 b$ représentent l'évolution pendant une impulsion.

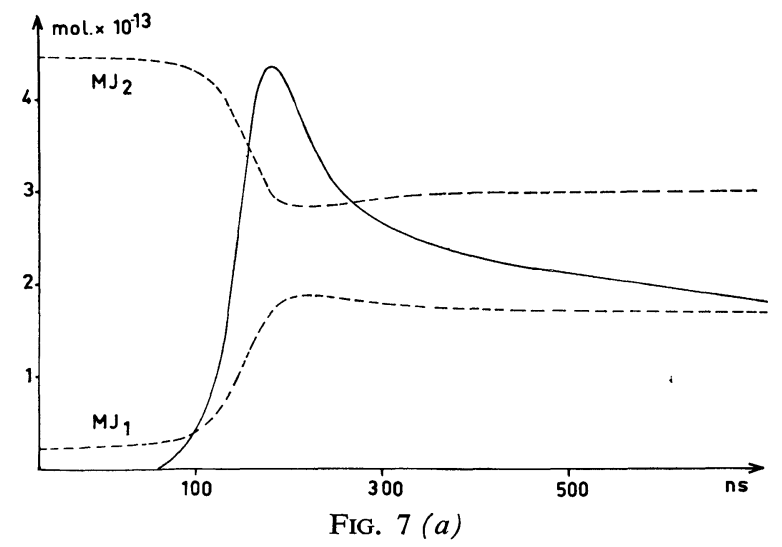

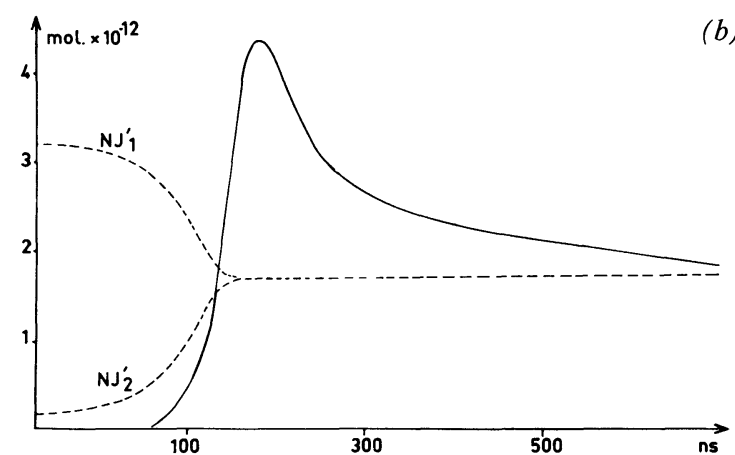

Fig. 7. - Evolution, pendant la durée d'une impulsion, des populations des niveaux rotationnels : a) Dans l'amplificateur. b) Dans l'absorbant saturable.
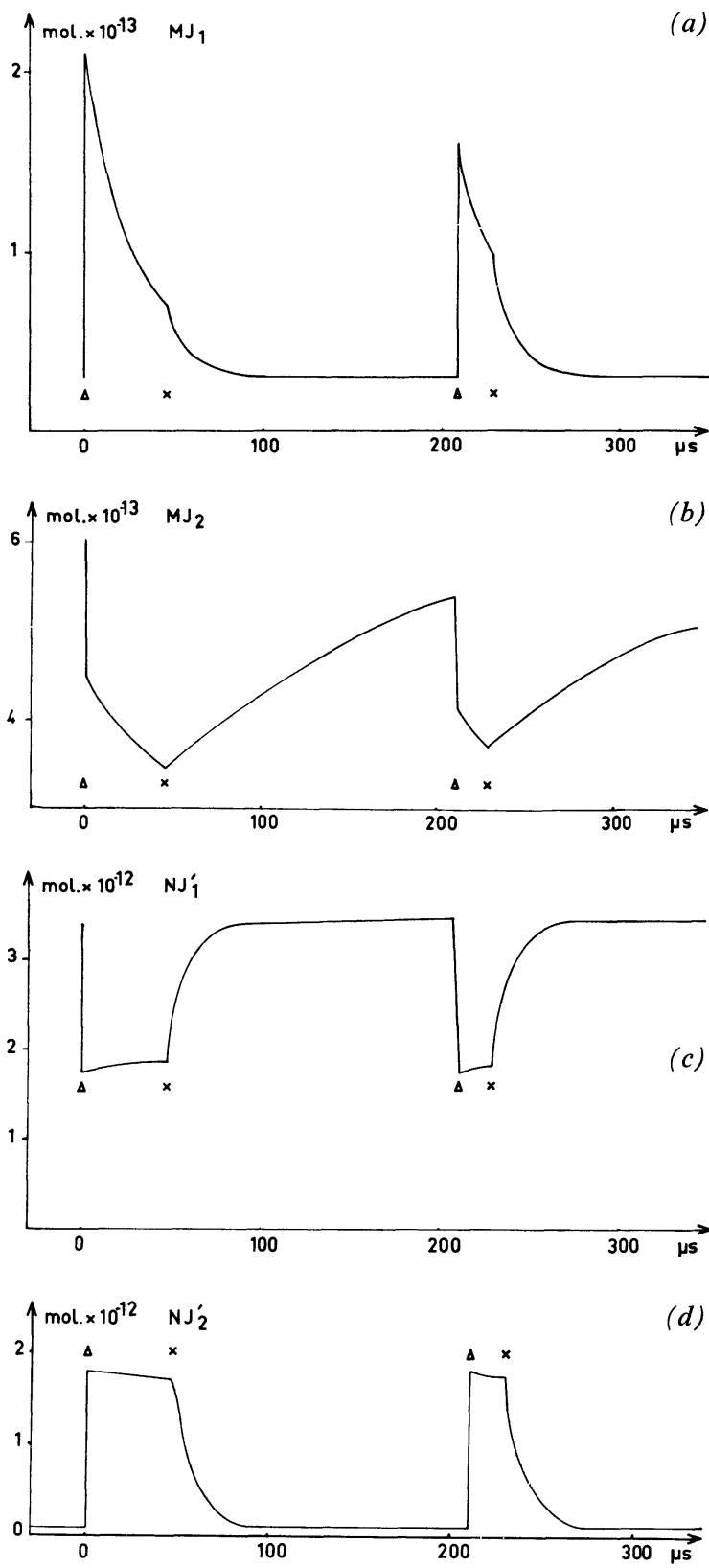

FIG. 8. - Evolution des populations des niveaux rotationnels inférieurs et supérieurs : $a$ et $b$ dans l'amplificateur ; $c$ et $d$ dans l'absorbant saturable ( $\Delta$ début de l'impulsion), ( $\times$ fin de la traîne). 
Au départ, l'absorbant est dans son état d'équilibre thermodynamique (absorption maximale). Le milieu amplificateur est en état d'amplification maximale et les populations $M_{\mathrm{J}_{1}}$ et $M_{\mathrm{J}_{2}}$ sont à leur valeur stationnaire $M_{\mathrm{J}_{1}}^{*}$ et $M_{\mathrm{J}_{2}}^{*}$. D'autre part la densité de photons est pratiquement nulle : il n'y a pas d'effet laser. Cette situation se rencontre si un écran opaque est placé dans la cavité. Si on retire cet écran, l'effet laser se construit. Dès que la densité de photons atteint un niveau suffisant, on constate que $M_{\mathrm{J}_{2}}$ diminue et que $M_{\mathrm{J}_{1}}$ augmente ce qui entraîne une diminution de l'amplification. De même $N_{\mathrm{J}_{1}^{\prime}}$ et $N_{\mathrm{J}_{2}^{\prime}}$ évoluent très rapidement, l'absorbant devient transparent. Donc le gain global augmente et l'énergie laser croît de plus en plus vite jusqu'au moment où l'amplification atteint un minimum qui est inférieur aux pertes fixes de la cavité. L'impulsion a atteint son sommet et va s'éteindre puisque les pertes surpassent le gain. Remarquons qu'à cet instant, l'absorbant est entièrement transparent. La décroissance s'amorce mais sera freinée par une remontée de $M_{\mathrm{J}_{2}}$ et une légère rechute de $M_{\mathrm{J}_{1}}$ qui sont dues à la relaxation rotationnelle du gaz carbonique. L'énergie laser décroît alors très lentement : c'est la traîne de l'impulsion. Pendant tout ce temps, les pertes excèdent très peu l'amplification. L'absorption augmente très lentement. Puis les pertes vont l'emporter nettement sur le gain et le laser s'éteint. Pendant l'extinction, $N_{\mathrm{J}_{1}^{\prime}}$ et $N_{\mathrm{J}_{2}^{\prime}}$ reviennent à leur état d'équilibre. $M_{\mathrm{J}_{1}}$ revient également très vite à sa valeur station- naire $M_{\mathrm{J}_{1}}^{*}$. Sous l'action du pompage, $M_{\mathrm{J}_{2}}$ augmente jusqu'à ce que le phénomène laser puisse s'amorcer alors que la valeur $M_{\mathbf{J}_{2}}^{*}$ n'est pas encore atteinte. L'impulsion suivante se déclenche alors, mais pour cette raison, elle n'atteint pas la puissance crête de la première. Le cycle se reproduit et toutes les impulsions suivantes sont identiques à la seconde. Dans un régime impulsionnel stable, on n'observe jamais la première impulsion. C'est pourquoi dans nos calculs nous utilisons toujours la seconde impulsion comme représentation de la réalité.

L'étude que nous avons entreprise du déclenchement passif, nous a permis de préciser l'importance des différents phénomènes de relaxation dans l'absorbant et dans l'amplificateur.

Grâce au programme de reconstitution que nous avons mis au point, nous avons la possibilité d'atteindre les valeurs des paramètres intervenant dans le déclenchement passif provoqué par un absorbant saturable. Ainsi nous avons déterminé la constante de relaxation rotationnelle dans $\mathrm{SF}_{6}$.

Cette méthode de calcul est utilisée actuellement dans le cas d'autres molécules telles que $\mathrm{CH}_{3} \mathrm{Cl}$, $\mathrm{CH}_{3} \mathrm{Br}, \mathrm{C}_{3} \mathrm{H}_{4}, \mathrm{CO}_{2}$ chauffé, avec lesquelles nous avons réalisé des expériences de déclenchement passif.

D'autre part, le programme sera complété afin de reconstituer des impulsions délivrées par un TEA laser et de prévoir leur amplification par des plasmas.

\section{Bibliographie}

[1] Wood, O. R., Schwarz, S. E., Appl. Phys. Lett. 11 (1967) 88-89.

[2] Karlov, N. V., Kuz'min, G. P., Petrov, Yu. N., ProKHOROV, A. M., JETP Lett. 7 (1968) 134-136.

[3] Burak, I., Houston, P., Sutton, D. G., Steinfeld, J. I., IEEE J. Quantum Electron., QE 72 (1971) 73-82.

[4] Flynn, G. W., Kovacs, M. A., Rhodes, C. K., Javan, A., Appl. Phys. Lett. 8 (1966) 63-65.

[5] Szabo, A., Stein, R. A., J. Appl. Phys. 36 (1965) 15621566.

[6] Hofelich-Abate, E., Hofelich, F., J. Appl. Phys., 39 (1968) 4823-4827.

[7] Lacome, N., Boulet, C., Arié, E., Can. J. Phys. 51 (1973) 302-310.

[8] Gray, L. D., Selvidge, J. E., J. Q. S. R. T. 5 (1965) 291301.

[9] Chéo, P. K., Abrams, R. L., Appl. Phys. Lett. 14 (1969) 47-49.

[10] LÉCUYER, A., Thèse Université Paris VI (1974).
[11] Jacobs, RR., Pettipiece, K. J., Thomas, S. J., Appl. Phys. Lett. 24 (1974) 375-377.

[12] Bailly, D., Gaultier, D., Rossetti, C., Barchewitz, P., Can. J. Phys. 50 (1972) 2605-2613.

[13] Steinfeld, J. I., Burak, I., Sutton, D. G., NowaK, A. V., J. Chem. Phys., 52 (1970) 5421-5434.

[14] Patel, C. K. N., Slusher, R. E. S., Phys. Rev. Lett. 18 (1967) 1019-1022.

[15] Burak, I., Steinfeld, J. I., Sutton, D. G., J. Q. S. R. T. 9 (1969) 959-980.

[16] Nhigan, W. L., Phys. Rev., A-2 (1970) 1989.

[17] Sharma, R. D., Phys. Rev. 177 (1969) 102.

[18] BuraK, I., Noter, Y., SzöKe, A., IEEE J. Quantum Electron. QE 9 (1972) 541.

[19] Bulthuis, K., Ponsen, G. J., IEEE J. Quantum Electron. QE 8 (1972) 597.

[20] Stark, E. E. Jr, Appl. Phys. Lett. 23 (1973) 335.

[21] Meyer, F., Dupré, J., MeYer, C., J. Mol. Spectros. 55 (1975) 28-30. 